\title{
Sinusoidally Modulated Silicon Leaky Wave Antenna for sub-THz Applications
}

\author{
Basma M. Yousef ${ }^{1}$, Allam M. Ameen ${ }^{2}$, Ahmed M. Attiya ${ }^{2}$, and A. A. Shaalan ${ }^{3}$ \\ ${ }^{1}$ Communications Department, Delta Higher Institute for Engineering and Technology, Mansoura, Egypt \\ ${ }^{2}$ Microwave Engineering Dept., Electronics Research Institute, Cairo, Egypt \\ ${ }^{3}$ Electronics and communications Department, Faculty of Engineering, Zagazig University, Zagazig, Egypt \\ *corresponding author, E-mail: basmamyousef@gmail.com
}

\begin{abstract}
A new sub-THz leaky wave antenna based on grounded silicon substrate for chip-to-chip communication applications is presented. The proposed leaky wave antenna is based on sinusoidally modulated surface reactance. This surface reactance is implemented by using varying width strips on the top of a grounded silicon substrate. Two approaches for developing these strips are studied. These two approaches are based on either highly doped silicon strips or gold strips. Both the doped silicon and the gold are presented by using their corresponding Drude models at the proposed operating frequency. Comparisons between the properties of these two approaches are presented to show the applicability of each one.
\end{abstract}

\section{Introduction}

The development of modern wireless communication systems requires a wide frequency bandwidth. Millimeter waves (mm-waves) and sub- $\mathrm{THz}$ frequencies have received much attention due to their applications such as short range radar system, high resolution beam-scanning imaging application, 5G cellular network, emerging sensing applications, and chip-to-chip communication [1]-[3]. Leaky wave antennas (LWAs) are travelling wave antennas which can be designed to perform beam scanning at mm-wave frequencies. Some of the benefits which make leaky wave antennas a promising solution for microwave and mm-wave applications are low cost, high directivity, high efficiency, simple feeding, and frequency beam scanning capability [4], [5]. Leaky wave antennas based on silicon substrate are good candidates for mm-wave applications [6]-[9]. This technology provides a low-cost platform for implementing high performance beam steering system at mm-wave frequencies. Integrating antenna system on a silicon substrate with other mm-wave components and circuits is a key technology for the mass market emerging mm-wave and sub$\mathrm{THz}$ communication systems.

A wide variety of LWAs were introduced, discussed and developed for microwave and mm-wave applications [10][12]. Planar printed LWAs are suitable for microwave range of frequencies [13]. Electromagnetic propagation along a sinusoidally modulated reactance surface was theoretically investigated as a technique to increase the gain of surfacewave antennas [14]. This sinusoidally modulated reactance surface is implemented by different techniques like varying width conducting strips on a grounded dielectric slab. Another example of a sinusoidally modulated mm-wave LWA based on inset dielectric waveguide is discussed in [15]. This antenna consists of a sinusoidally modulated inset groove etched on a dielectric rectangular waveguide. In [16] another $\mathrm{THz}$ sinusoidally modulated leaky wave is introduced based on tunable modulated graphene sheet. In this case, the antenna consists of a graphene ribbon modulated by adjusting its chemical potential by using applied spatially varying electrostatic field.

On the other hand, highly doped silicon is found to have plasmonic properties at THz and far infrared range [17], [18]. This property makes highly doped silicon a good candidate for nanoantenna applications at this frequency range. In addition, highly doped silicon can be considered as a conducting layer on the silicon substrate. Developing highly doped silicon can be obtained by using the same technology of developing active components on a silicon substrate. In addition, active silicon components are found to be suitable for mm-wave circuits in different mm-wave and sub- $\mathrm{THz}$ applications [19], [20]. This is the motivation in this paper to introduce the idea of using highly doped silicon to integrate a sinusoidally modulated leaky wave antenna with sub-THz active circuits. This configuration can be quite useful for chip-to-chip communication [1], [21].

In this paper, the analysis and design of two different configurations of sinusoidally modulated silicon LWA for $300 \mathrm{GHz}$ chip-to-chip communication are presented. The first configuration is based on using highly doped silicon strips on a grounded silicon substrate and the second configuration is based on gold strips on a grounded silicon substrate.

The equivalent surface reactance of the conducting periodic strips on a grounded dielectric slab is obtained by studying the reflection coefficient of infinite grating structure composed of these strips. This equivalent reactance depends on the width and the periodicity of these conducting strips. Thus, it would be possible to tailor the required profile of equivalent surface reactance by using multi-width conducting strips. 
In Sec. II the electrical properties of highly doped silicon are presented. Then in Sec. III, the corresponding properties of gold are also discussed. The design steps of a sinusoidally modulated reactance leaky wave antenna are discussed briefly in Sec. IV. The electrical properties of doped silicon and gold discussed in Sec. II and III are used to study the equivalent surface reactance of a grounded silicon substrate loaded by periodic array of strips of either doped silicon or gold in Sec. V. This study is used to determine the widths of the strips along the cell of the proposed leaky wave antenna according to the required reactance profile obtained in Sec. IV. The designed cells in Sec. V are combined in Sec. VI to introduce the complete sinusoidally modulated leaky wave antenna structure. Two designs are presented; one is based on highly doped silicon strips and the other is based on gold strips. Results and discussions of these two designs are presented and compared. The analysis and design of these antenna configurations are performed using HFSS and is compared with CST.

\section{THz conductivity of highly doped silicon}

The electrical properties of heavily doped silicon were studied extensively by different methods [22], [23]. A recent model for the conductivity of doped silicon was presented as a generalized Drude model (GD-model) as follows [23]:

$$
\sigma_{\text {doped_Si }}(\omega)=\frac{\sigma(0)}{\left[1-\left(-j \omega \tau_{S i}\right)^{1-\alpha}\right]^{\beta}}
$$

where $\alpha, \beta$ are real numbers as $\alpha \geq 0, \beta \leq 1, \sigma(0)$ is the dc conductivity, $\omega$ is the angular frequency and $\tau_{S i}$ is the corresponding scattering time. These parameters depend on the concentration of doping density. For a highly doped silicon with doping concentration $n_{o}=10^{18} \mathrm{~cm}^{-3}$, the parameters of this generalized Drude model are $\alpha=0.19$, $\beta=0.82, \tau_{S i}=0.071 \mathrm{ps}$ and $\sigma(0)=42.17 \mathrm{~S} / \mathrm{cm}$ [23]. In this case, the complex conductivity of doped silicon as a function of frequency is obtained for high doping density as shown in Fig. 1. The real conductivity of highly doped silicon at $300 \mathrm{GHz}$ is of order $3950 \mathrm{~S} / \mathrm{m}$ while the corresponding imaginary part is of order $600 \mathrm{~S} / \mathrm{m}$.

\section{THz conductivity of thin gold film}

In a similar way, the conductivity of thin gold film on a silicon substrate can be represented by using Drude model as follows [24]:

$$
\sigma_{A u}(\omega) \cong \frac{\varepsilon_{0} \omega_{p}^{2} \tau_{A u}}{\left[1+j \omega \tau_{A u}\right]}
$$

where $\omega_{p} / 2 \pi$ for films of thickness greater than $20 \mathrm{~nm}$ is $2080 \mathrm{THz}, \tau_{A u}=18 \mathrm{fs}$. Figure 2 shows the real and imaginary conductivity parts of the thin gold film in sub-THz range. It can be noted that the real conductivity in this case of order $2.7 \times 10^{7} \mathrm{~S} / \mathrm{m}$ which is much greater than the real conductivity of the highly doped silicon. On the other hand, the imaginary conductivity part of the thin gold is of order $8 \times 10^{5} \mathrm{~S} / \mathrm{m}$. It can be noted that the ratio of the imaginary part of conductivity to the real part in the case of gold is much smaller that the corresponding ratio in doped silicon. Thus, in the case of gold, the wave propagation depends mainly on the real part conductivity.
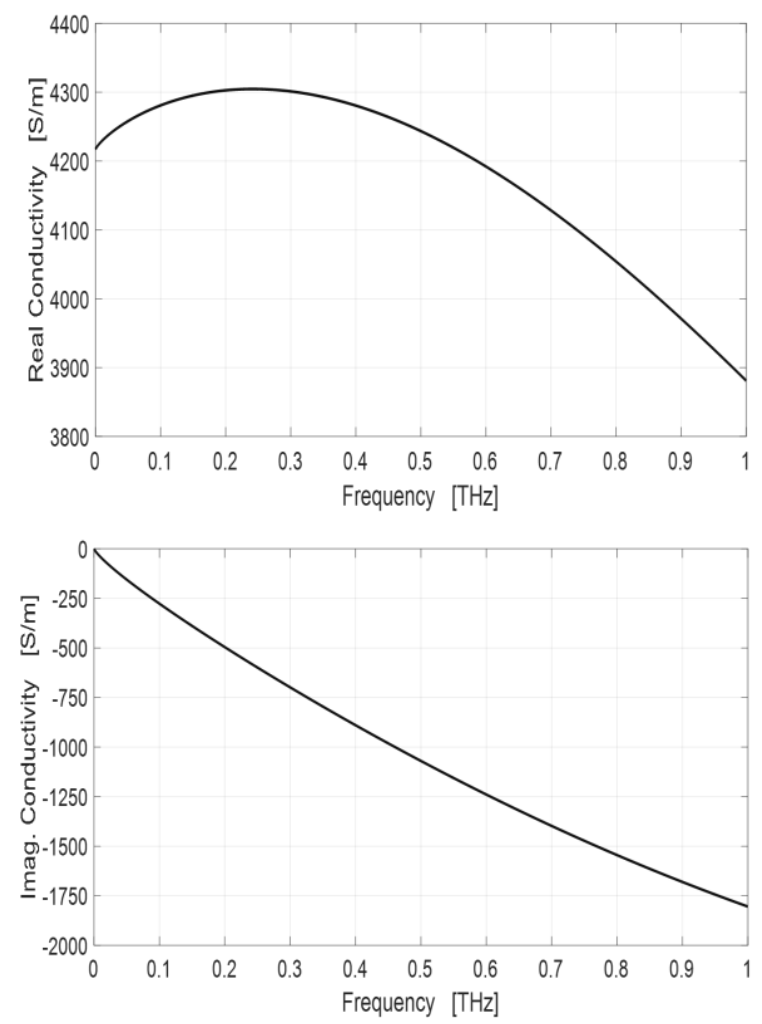

Figure 1: The variation of the complex conductivity (real part and imaginary part) as a function of the frequency for high doping concentration $\left(n_{o}=10^{18} \mathrm{~cm}^{-3}\right)$.
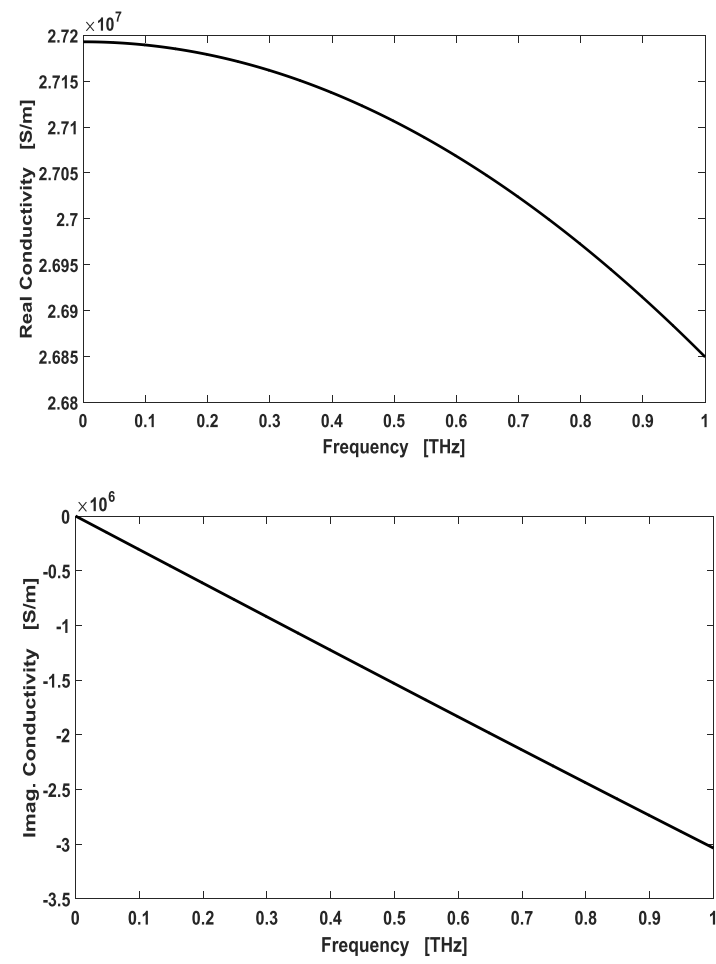

Figure 2: The variation of the complex conductivity (real part and imaginary part) as a function of the frequency for a thin gold film on a silicon substrate. 


\section{Design of sinusoidally modulated reactance surface leaky wave antenna}

In sinusoidally modulated reactance surface, the reactance of the surface varies periodically in sinusoidal form along the longitudinal propagation axis (y-axis in the present case). This variation in surface reactance introduces leaky wave radiation [14]. Sinusoidally modulated reactance surface can be presented as:

$$
Z_{\text {surf }}(y)=j \eta_{0} X^{\prime}\left[1+M \cos \left(\frac{2 \pi y}{a}\right)\right]
$$

where $Z_{\text {surf }}(y)$ is the surface impedance along the $\mathrm{y}$ direction, $X^{\prime}$ is the average normalized surface reactance, $\eta_{0}=120 \pi \Omega$ is the free space characteristic impedance, $M$ is the modulation factor and $a$ is the periodic length. This sinusoidally modulated reactance surface supports propagation of leaky waves. The complex propagation constant $k_{y}$ of this leaky wave along this modulated reactance surface can be obtained approximately for small modulation factor as follows [16]:

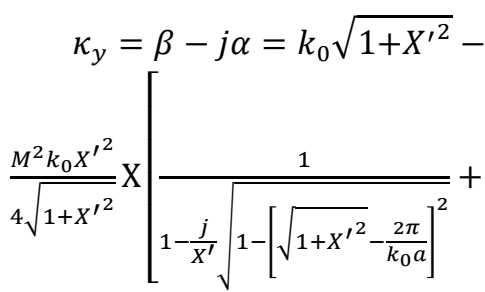

$\frac{1}{\left.1-\frac{j}{X^{\prime}} \sqrt{1-\left[\sqrt{1+X^{\prime 2}}+\frac{2 \pi}{k_{0} a}\right]^{2}}\right]}$

where $\beta$ is the real propagation constant along the leaky wave antenna and $\alpha$ is the attenuation coefficient. The direction of the radiated leaky wave beam depends on $\beta$ while the radiation beam width depends on $\alpha$. According to the approximation of (4), $\beta$ is mainly depending on the average normalized surface reactance $X^{\prime}$. On the other hand, the attenuation coefficient $\alpha$ is proportional to the modulation index $M$. Thus, the radiated beam width increases by increasing the modulation index.

The relation between the angle of the main beam and both $X^{\prime}$ and $a$ can be obtained by simplifying (4) as follows [15]:

$$
\sin \left(\theta_{n=-1}\right) \approx \sqrt{1+X^{\prime 2}}-\frac{2 \pi}{k_{0} a}
$$

For the present analysis, the average normalized reactance surface is assumed to be $X^{\prime}=1.2$, the operating frequency is $300 \mathrm{GHz}$ and the required beam direction is $\theta=$ $30^{\circ}$. For these parameters, the periodic length $a$ can be obtained from (5) as $a=940 \mu \mathrm{m}$. In the present design, the modulation factor $M$ is assumed to be 0.2 .

This modulated surface reactance cell of length $a$ is divided into smaller sub-cells of periodic conducting strips. The spacing between the centers of adjacent strips is assumed to be $a / 10$. The local equivalent surface reactance of each cell is obtained by adjusting the width of this strip. The equivalent surface reactance for a specific width can be obtained by studying the reflection of an infinite array of strips of this width with the proposed periodicity between the adjacent strips. The equivalent surface reactance is obtained as a function of the strip width. Then by using the required surface reactance profile on the modulated surface reactance cell one can obtain the corresponding width of each strip in the sub-cells. This modulated surface reactance surface is repeated periodically with a periodic length $a$ to introduce the leaky wave structure. In the present antenna, eight modulated surface reactance surface cells are used to construct the required leaky wave structure. This leaky wave structure is connected to two tapered sections at its two ends to be matched with the feeding transmission line. These steps are discussed in the following two sections.

\section{Analysis of the equivalent reactance surface}

The proposed antenna is based on a grounded silicon substrate with a thickness $60 \mu \mathrm{m}$ and a dielectric constant $\varepsilon_{r}=11.9$. This grounded silicon substrate is assumed to be loaded by an infinite array of conducting strips. These conducting strips are studied here for both highly doped silicon strips and gold strips. For highly doped silicon, the depth of high doping is less than or equal to $3 \mu \mathrm{m}$ according to the doping technique [22].

The equivalent reactance surface is composed of periodic cells of periodic length $p=0.1 a=94 \mu \mathrm{m}$. The width of the strips is varied from $w=10 \mu \mathrm{m}$ to $90 \mu \mathrm{m}$. The equivalent reactance is obtained by using the reflection of normally incident plane wave on these periodic cells as follows:

$$
X=\frac{\operatorname{im}\left(z_{\text {surf }}\right)}{\eta_{0}}=\operatorname{im}\left(\frac{1+\Gamma}{1-\Gamma}\right)
$$

where $\Gamma$ is the reflection coefficient of normally incident plane wave from this surface. This reflection coefficient of an infinite periodic structure can be obtained by simulating only a single cell with periodic boundary conditions as shown in Fig. 3.

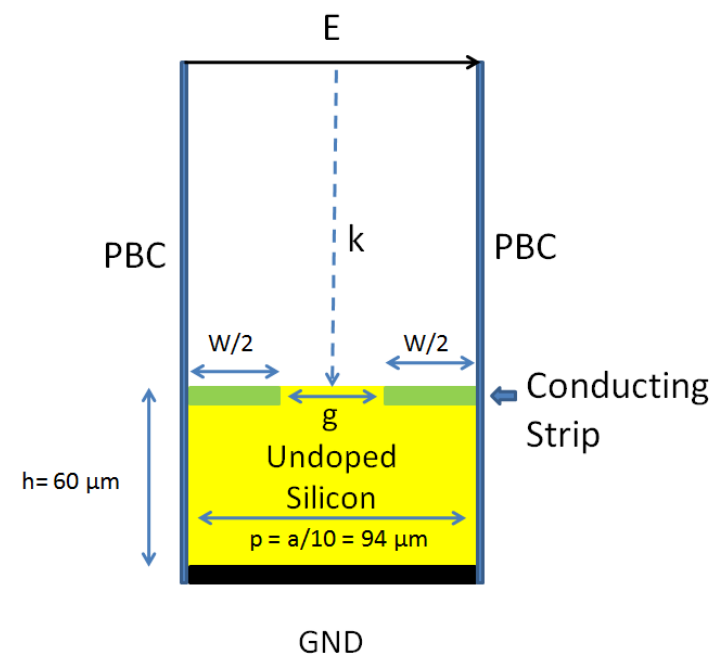

Figure 3: Geometry of a unit cell in a grounded silicon slab loaded by infinite periodic conducting strips. 
Figure 4 shows the normalized equivalent reactance surface of the unit cell as a function of the width of the doped strips for both highly doped silicon strips and gold strips.

The required unit cell of the proposed leaky wave antenna consists of ten strips as shown in Fig. 5. The length of the each strip is assumed to be $750 \mu \mathrm{m}$. The surface reactance of this unit cell is varied according to (3) with the parameters $X^{\prime}, a$ and $M$ discussed in Sec. IV. The width of each strip is determined according to the required surface reactance at the center position of this strip. This is done by using obtained relation between the equivalent surface reactance and the width of the strip shown in Fig. 4. Since the unit cell is symmetric, only half the cell is determined and the other half is obtained as a symmetric image. This procedure was followed to determine the required values of surface reactance and the corresponding widths as shown in Table 1.

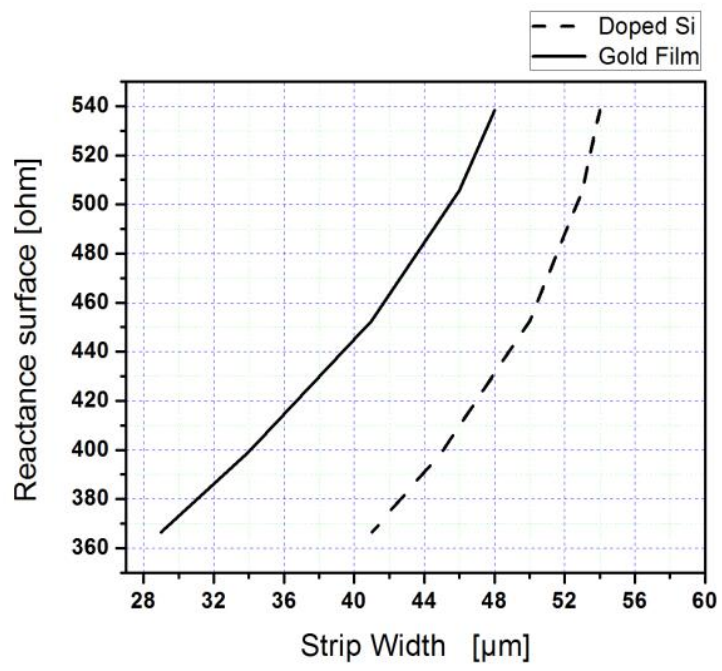

Figure 4: Equivalent surface reactance of a grounded silicon substrate of thickness $60 \mu \mathrm{m}$ loaded by periodic strips of width $\mathrm{W}$ and periodic distance $\mathrm{p}=94 \mu \mathrm{m}$.

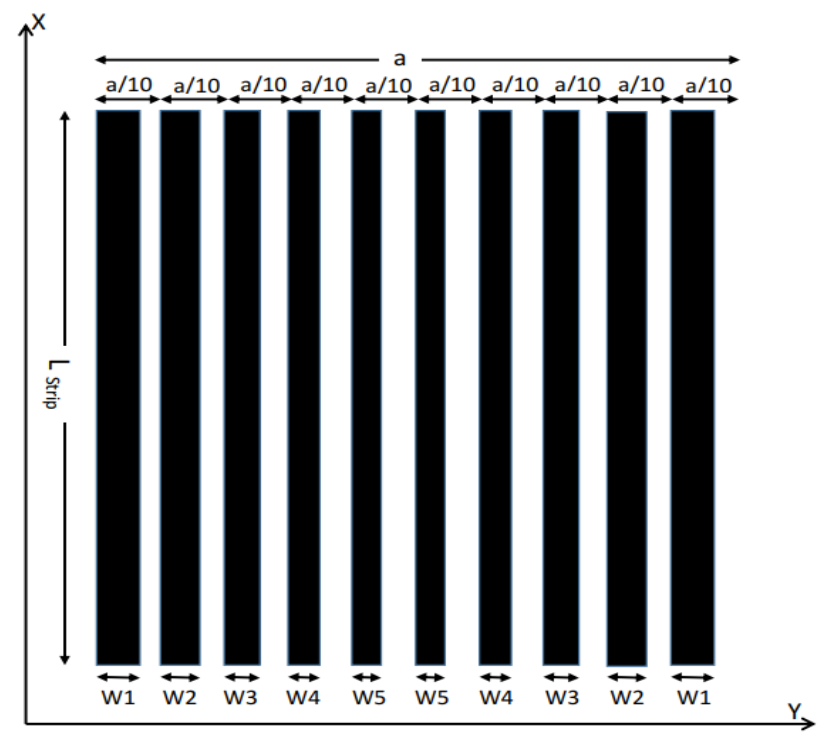

Figure 5: Geometry of a unit cell of the proposed sinusoidally modulated leaky wave antenna.
Table 1: Width distribution of both the doped silicon and gold film to introduce sinusoidally modulated reactance surface as shown in Fig. 5.

\begin{tabular}{|c|c|c|c|}
\hline Strip \# & $\begin{array}{c}\text { Required } \\
\text { surface } \\
\text { reactance } \\
\text { X surface }(\boldsymbol{\Omega})\end{array}$ & $\begin{array}{c}\text { Width of } \\
\text { strip }(\boldsymbol{\mu m}) \\
\text { for doped } \\
\text { silicon }\end{array}$ & $\begin{array}{c}\text { Width of } \\
\text { strip }(\boldsymbol{\mu m}) \\
\text { for gold } \\
\text { film }\end{array}$ \\
\hline W1 & 538.44 & 54 & 48 \\
\hline W2 & 505.57 & 53 & 46 \\
\hline W3 & 452.39 & 50 & 41 \\
\hline W4 & 399.21 & 45 & 34 \\
\hline W5 & 366.34 & 41 & 29 \\
\hline
\end{tabular}

\section{Analysis of the complete leaky wave antenna}

In this section two configurations of a sinusoidally modulated leaky wave antenna are presented. Each antenna consists of eight unit cells. The first configuration is based on using strips of highly doped silicon with doping concentration $n_{o}=10^{18} \mathrm{~cm}^{-3}$ and the second one is based on gold strips. The width of each cell is $a=940 \mu \mathrm{m}$ and the length of each strip is $750 \mu \mathrm{m}$. The leaky wave antenna structure is terminated by two tapered line sections to match its ports to $50 \Omega$ feeding line. The length of this section is assumed to be $1000 \mu \mathrm{m}$. The proposed antenna structure of a sinusoidally modulated leaky wave antenna is shown in Fig. 6. This antenna is designed to operate at $300 \mathrm{GHz}$ with a scanning angle $30^{\circ}$. The total antenna dimension is $1.75 \times 9.55 \mathrm{~mm}^{2}$.

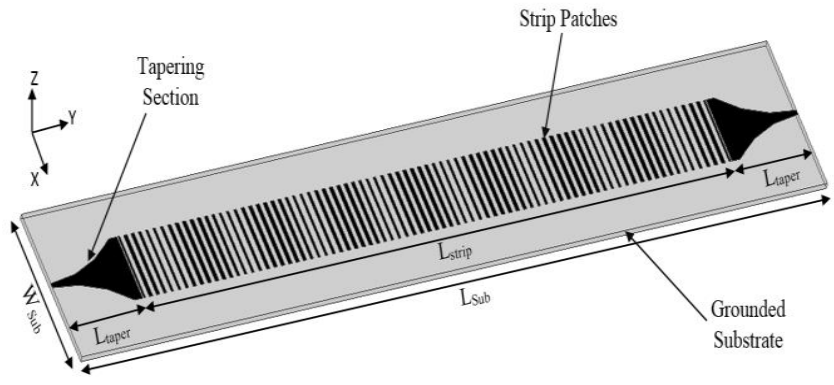

Figure 6: Geometry of the proposed sinusoidally modulated leaky wave antenna structure.

Figure 7 shows the transmission and reflection coefficients for these two antenna configurations. It can be noted that due to the dissipative properties of highly doped silicon, the input reflection coefficient is nearly linear along the operating frequency band. However, for gold strips, the reflection coefficient is characterized by clear resonance behavior at the design operating frequency of $300 \mathrm{GHz}$. The dissipative properties of doped silicon is also the reason for reducing the transmission coefficient compared with the corresponding one of the gold strips.

The corresponding radiation efficiency is presented in Fig. 8. It is quite clear that the dissipative properties of doped silicon antenna has a significant effect on reducing the radiation efficiency compared with gold strips antenna. However, this dissipative property makes the matching and antenna efficiency slowly varying across a wider bandwidth. 
According to the specification of the required communication link, one can choose the appropriate antenna configuration.

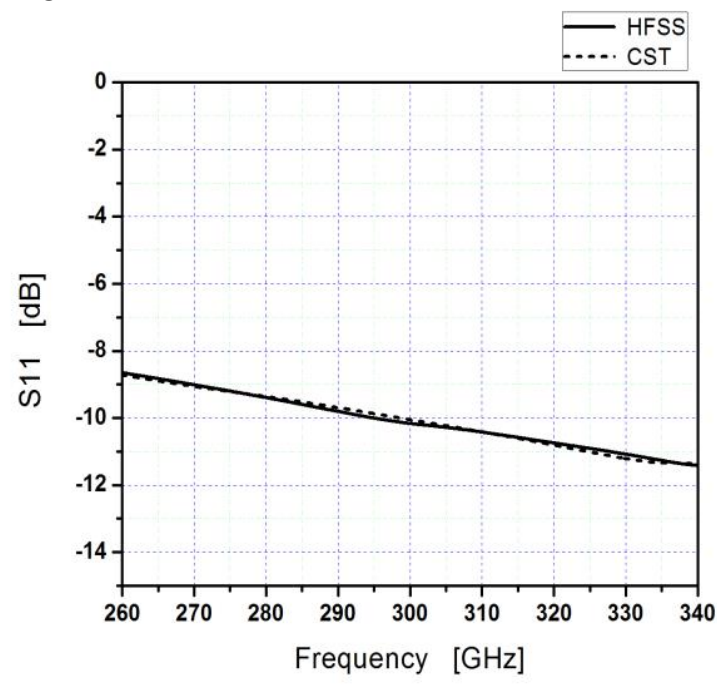

(a)

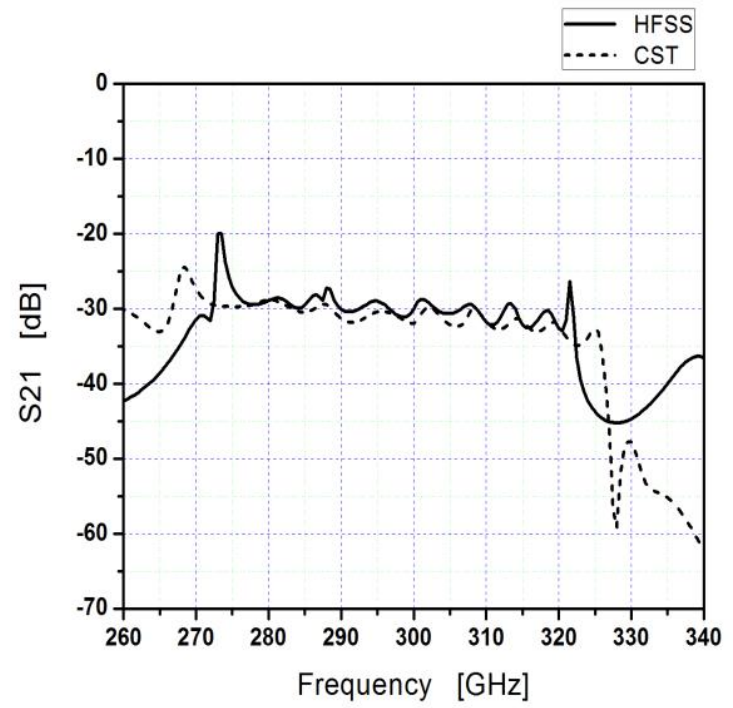

(b)

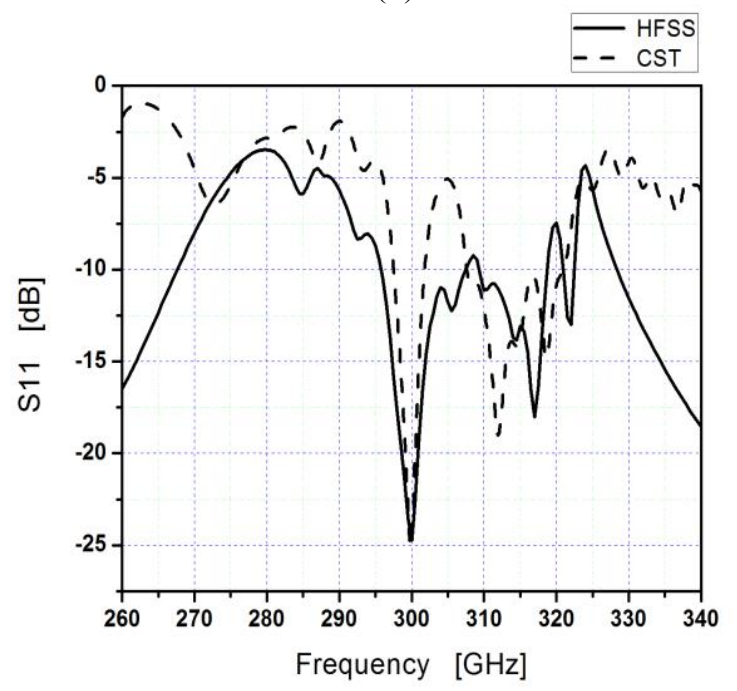

(c)

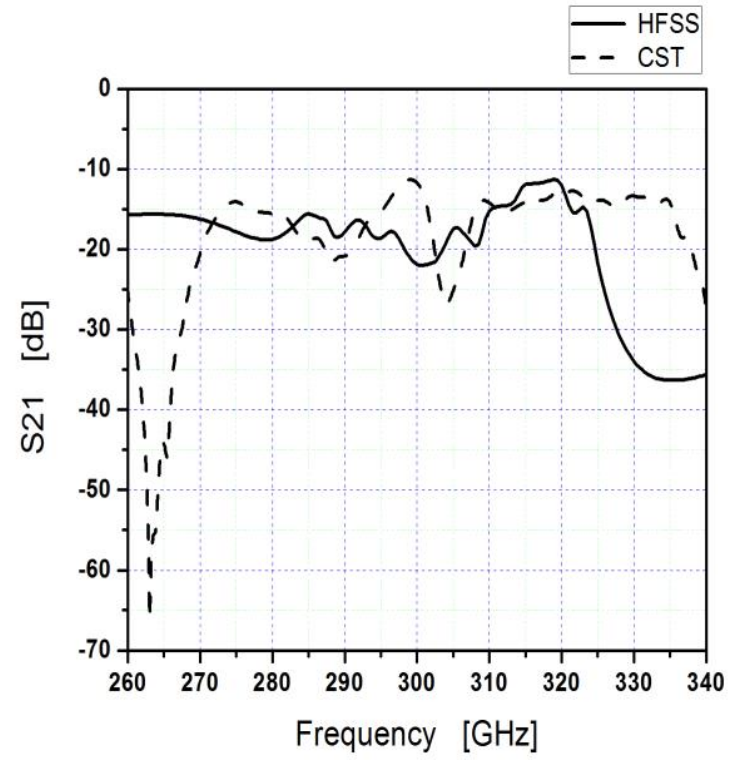

(d)

Figure 7: Reflection and transmission coefficients of the designed sinusoidally modulated leaky wave antennas. (a)reflection coefficient of silicon doped antenna, (b)transmission coefficient of silicon doped antenna, (c)reflection coefficient of gold strips antenna and (d)transmission coefficient of gold strips antenna.

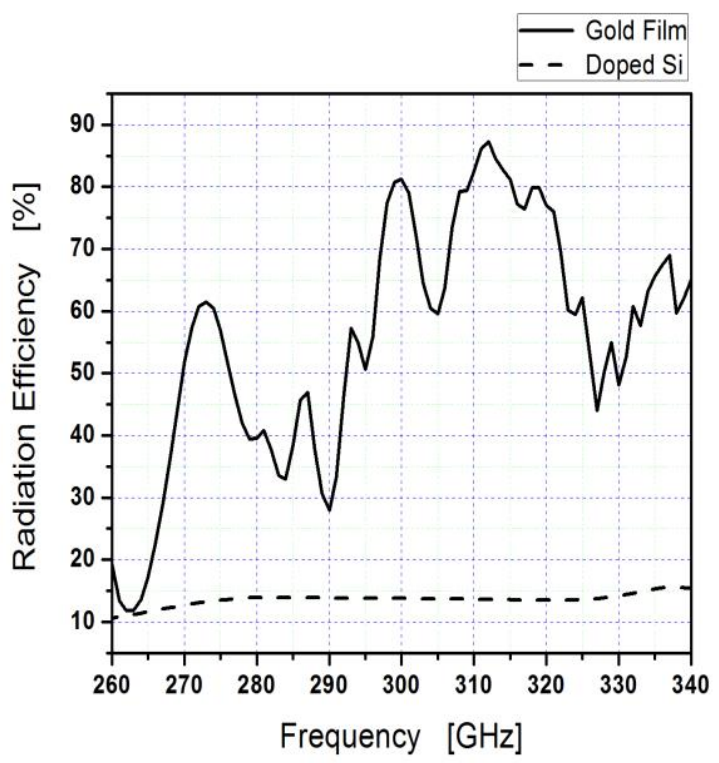

Figure 8: The radiation efficiency of the designed sinusoidally modulated leaky wave antennas.

Finally, Fig. 9 shows the obtained antenna gain (values in ratio) along the E-plane of the antenna configurations at $300 \mathrm{GHz}$. Both antennas satisfy the peak radiation at the design scanning angle of $30 \mathrm{o}$. However, the highly doped silicon antenna has much less peak gain compared with the gold strips antenna. The reason for this is the low efficiency of the doped silicon antenna due to the corresponding dissipative properties. 


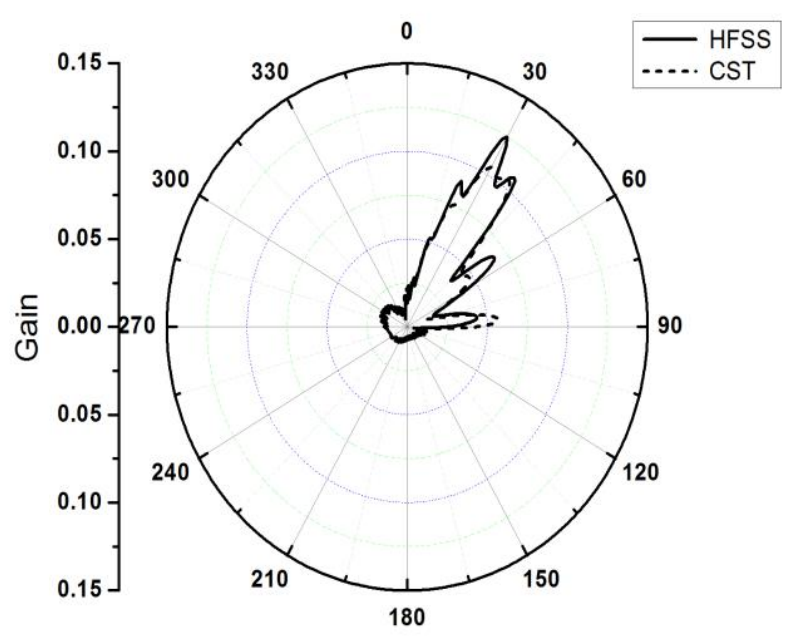

(a)

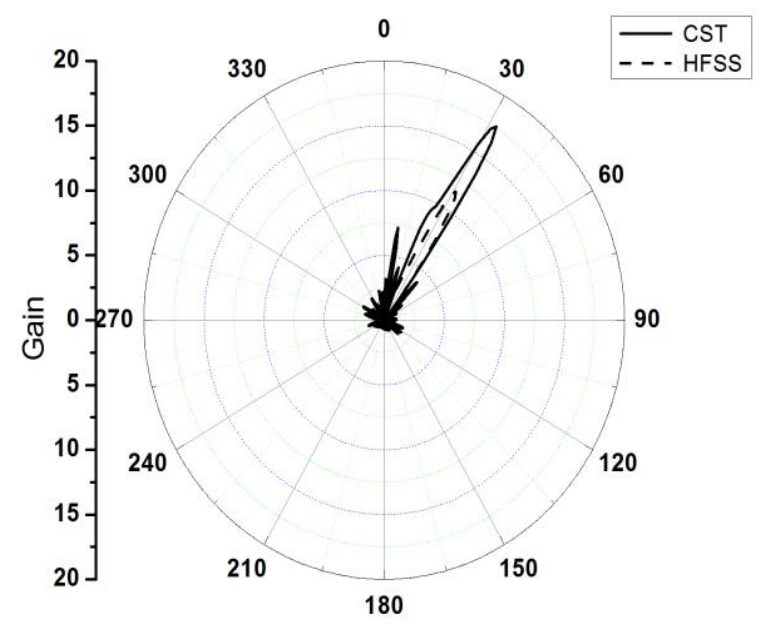

(b)

Figure 9: Gain (ratio) along E-plane at $f_{0}=300 \mathrm{GHz}$, (a) for doped silicon, and (b) for a thin gold film.

\section{Conclusions}

In this paper, a sinusoidally modulated reactance surface leaky wave antenna on a grounded silicon substrate is discussed. The proposed antenna is designed to operate at a frequency of $300 \mathrm{GHz}$ with a beam pointed to $30^{\circ}$ for chipto-chip communication. The analysis and design of the proposed antenna is presented in detail. The sinusoidally modulated reactance surface is implemented using varying width strips array above the grounded dielectric silicon substrate. Two configurations for these strips are introduced based on either highly doped silicon strips or gold strips. The antenna configuration based on highly doped silicon strips shows dissipative properties which introduce low efficiency and low antenna gain. However, this configuration is characterized by wideband matching properties and slowly varying radiation properties in a wide frequency band. On the other hand, the antenna configuration based on gold strips shows higher efficiency and higher antenna gain. However, this configuration is characterized by narrow band resonance behavior around the operating design frequency. According to the requirements of the proposed system, one can choose between wide band low efficiency antenna with doped silicon strips or narrow band high efficiency antenna with gold strips.

\section{References}

[1] Hwangbo, Seahee, Yong-Kyu Yoon, and Aric B. Shorey, "Millimeter-wave wireless chip-to-chip (c2c) communications in $3 \mathrm{~d}$ system-in-packaging (sip) using compact through glass via (tgv)-integrated antennas," IEEE 68th Electronic Components and Technology Conference (ECTC), pp. 2074-2079, 2018.

[2] Yu, Xianbin, S. Jia, Hao Hu, Michael Galili, Toshio Morioka, Peter Uhd Jepsen, and Leif Katsuo Oxenlwe, "160 Gbit/s photonics wireless transmission in the 300$500 \mathrm{GHz}$ band," Apl Photonics 1, no. 8, 2016.

[3] Kleine-Ostmann, Thomas, and Tadao Nagatsuma, "A review on terahertz communications research." Journal of Infrared, Millimeter, and Terahertz Waves 32, no. 2, pp. 143-171, 2011.

[4] F. Schwering and S. T. Peng, "Design of dielectric grating antennas for millimeter-wave applications," IEEE Trans. Microwave Theory Tech., vol. MTT-31, pp. 199-209, February 1983.

[5] Zandieh, A.S. Abdellatif; A. Taeb, and S. SafaviNaeini, "Low-cost and high-efficiency antenna for millimeter-wave frequency scanning applications," IEEE Antennas and Wireless Propagation Letters, vol.12, no., pp.116-119, 2013.

[6] Aidin Taeb, Ahmed Shehata Abdellatif, Gholamreza Z. Rafi, Suren Gigoyan, Safieddin Safavi-Neini and Mohammad Basha, " A low-cost silicon-based beam-steering grating antenna for g-band applications," IEEE Antennas and Propagation Society International Symposium (APSURSI), pp. 1282 - 1283, 2014.

[7] Karim Tekkouk, Jiro Hirokawa, Kazuki Oogimoto, Tadao Nagatsuma, Hiroyuki Seto, Yoshiyuki Inoue and Mikiko Saito, " Corporate-feed slotted waveguide array antenna at $350 \mathrm{GHz}$ band by silicon process," IEEE Antennas and Propagation Society International Symposium (APSURSI), pp. 1197 - 1198, 2016.

[8] Ramadan A. Alhalabi and Gabriel M. Rebeiz, "Design of high-efficiency millimeter-wave microstrip antennas for silicon rfic applications," IEEE Antennas and Propagation Society International Symposium (APSURSI), pp. 2055 - 2058, 2011.

[9] Peiqin Liu, Yue Li, Zhijun Zhang, Shaodong Wang and Zhenghe Feng, "A fixed-beam leaky-wave cavity backed slot antenna manufactured by bulk silicon mems technology," IEEE Transactions on Antennas and Propagation, Volume: 65 , Issue: 9, pp. $4399-4405$, 2017. 
[10] Oliner and R. C. Johnson, "Leaky-wave antennas", Antenna Engineering Handbook, McGraw-Hill, 1993.

[11] L. Liu, C. Caloz, and T. Itoh, "Dominant mode leakywave antenna with backfire-to-endfire scanning capability," Electronics Letters, vol.38, no.23, pp.14141416, 7 Nov 2002.

[12] F. Xu, K. Wu, and X. Zhang, "Periodic leaky-wave antenna for millimeter wave applications based on substrate integrated waveguide," IEEE Transactions on Antennas and Propagation, vol.58, no.2, pp.340-347, Feb. 2010.

[13] Z. Tianxia; D.R. Jackson, J.T. Williams, H.-Y.D. Yang; and A.A. Oliner, "2-D periodic leaky-wave antennaspart I: metal patch design," IEEE Transactions on Antennas and Propagation, vol.53, no.11, pp.3505 3514, Nov. 2005.

[14] A. A. Oliner and A. Hessel, "Guided wave on sinusoidally-modulated reactance surfaces," IRE Trans. Antennas Propagation., vol. 7, no. 4, pp. 201-208, Dec. 1959.

[15] Xue Bai, Shi-Wei Qu, Kung-Bo Ng and Chi Hou Chan, " Sinusoidally-modulated leaky-wave antenna for millimeter-wave application," IEEE Transactions on Antennas and Propagation, Volume: 64, Issue: 3, pp. $849-855,2016$.

[16] Yan Cheng, Lin-Sheng Wu, Min Tang, Yao-Ping Zhang and Jun-Fa Mao, "A sinusoidally-modulated leakywave antenna with gapped graphene ribbons," IEEE Antennas and Wireless Propagation Letters, Volume: 16, pp. 3000 - 3004, 2017.

[17] DiPippo, William, Bong Jae Lee, and Keunhan Park. "Design analysis of doped-silicon surface Plasmon resonance immunesensors in mid-infrared range." Optics express, Vol. 18, no. 18 pp.1939619406, 2010.

[18] Law, Stephanie, Lan Yu, Aaron Rosenberg, and Daniel Wasserman. "All-semiconductor plasmonic nanoantennas for infrared sensing." Nano letters Vol. 13, no. 9, pp. 4569-4574. 2013.

[19] Lacombe, E., C. Belem-Goncalves, C. Luxey, F. Gianesello, C. Durand, D. Gloria, and G. Ducournau, "300 GHz OOK transmitter integrated in advanced silicon photonics technology and achieving $20 \mathrm{~Gb} / \mathrm{s}$," IEEE Radio Frequency Integrated Circuits Symposium (RFIC), pp. 356-359, 2018.

[20] Fujishima, Minoru, "Key technologies for THz wireless link by silicon CMOS integrated circuits," In Photonics, vol. 5, no. 4, p. 50. Multidisciplinary Digital Publishing Institute, 2018.

[21] Kim, Seunghwan, and Alenka Zajić, "Characterization of $300-\mathrm{GHz}$ wireless channel on a computer motherboard," IEEE Transactions on Antennas and Propagation 64, no. 12, pp. 5411-5423, 2016.

[22] R. Chaoui, B. Mahmoudi, A. Messaoud, Y. Si Ahmed, A. Mefoued and B. Mahmoudi, "Phosphorus emitter profile control for silicon solar cell using the doss diffusion technique, " Revue des Energies Renouvelables, Vol. 19, pp. 303 - 309, 2016.

[23] K. J. Willis, S. C. Hagness, and I. Knezevic, "A generalized Drude model for doped silicon at terahertz frequencies derived from microscopic transport simulation, " Applied Physics Letters, Vol. 102, 2013.

[24] Walther, M., D. G. Cooke, C. Sherstan, M. Hajar, M. R. Freeman, and F. A. Hegmann, "Terahertz conductivity of thin gold films at the metal-insulator percolation transition," Physical Review B 76, no. 12, pp. 125408, 2007. 